\title{
Society of Skeletal Radiology 2009 annual meeting
}

\author{
Laura W. Bancroft
}

Published online: 23 August 2009

(C) ISS 2009

\begin{abstract}
Peer-reviewed abstracts presented at the 2009 Society of Skeletal Radiology (SSR) Annual Meeting were reviewed again following oral presentation. Topics representing new concepts, new or novel imaging techniques and instructive case series involving the musculoskeletal system, felt to be of potential interest to investigators and practicing clinicians, have been highlighted in this compilation and analysis.
\end{abstract}

Keywords Musculoskeletal imaging · Medical societies . Education $\cdot$ Research $\cdot$ New concepts

\section{Introduction}

The Society of Skeletal Radiology (SSR) held its 32nd annual meeting at the Wild Dunes Resort in the Isle of Palms, South Carolina, from 8 March through 11 March 2009. The meeting was attended by 237 society members.

\section{Scientific program}

This year's scientific program consisted of 44 abstracts selected by the program committee from those submitted by society members, reflecting their active clinical research. Abstracts were presented in eight major topic sessions, including tumor, knee, hip, shoulder, arthritis, ultrasound, upper and lower extremity, as well as basic research and techniques. Abstracts for the research session were selected by the society research committee on the basis of scientific

\section{W. Bancroft $(\bowtie)$}

Department of Radiology, Florida Hospital,

601 E. Rollins,

Orlando, FL 32803, USA

e-mail: laura.bancroft.md@flhosp.org content. Special interest topics were those that were notable, but not readily classified into other existing categories. A listing of the scientific program has been previously published [1]. Presented below is a summary of some of the meeting highlights.

\section{Meeting highlights}

While the general meeting abstracts were presented by subject matter, it is easier to summarize the meeting highlights by themes or emphasis, as noted below. As with past meetings, the largest number of abstracts addressed imaging appearances, followed by imaging protocols/techniques, imaging modalities, intervention, normal variations, and radiology education.

\section{Imaging appearances}

The imaging features of a number of specific lesions were addressed, including sports-related injuries, intra-articular pathology, expected postsurgical imaging findings, and tumors. A review of the clinical and magnetic resonance imaging (MRI) records of three professional ice hockey clubs yielded eight cases of acute syndesmotic ankle sprains [2]. A consistent pattern of tearing of the anterior inferior tibiofibular ligament (AITFL) with an intact anterior talofibular ligament (ATFL) was identified in these hockey players with 'high ankle sprain.' The mechanism of injury and the support of the hockey boot were postulated to protect the ATFL in these types of sports injuries.

The expected MRI appearance of the postoperative rotator cuff was delineated in a temporal evolution of 40 patients with full-thickness tears [3]. Preoperative, 6 week, 3 month and 12 month follow-up MR images were obtained and evaluated for the size of the tendon footprint, tendon 
thickness, signal intensity and presence/absence of re-tear. There was no significant difference in results between patients who had single or double row repair. The majority of tendon healing occurred between 3 months and 12 months, and there was a $10 \%$ re-tear rate. A thin tendon and a small postoperative footprint were not indicative of a poor prognosis. There was significant variability in tendon signal intensity, which did not correlate with functional outcomes.

The Armed Forces Institute of Pathology reported on their experience of 40 cases of pathologically proven telangiectatic osteosarcoma [4]. Characteristic imaging features included aggressive geographic bone destruction, subtle peripheral osteoid matrix and central cystic regions. This subtle osteoid matrix in the viable sarcomatous tissue about hemorrhagic or necrotic regions was detected on radiographs in $58 \%$ of cases and by computed tomography (CT) in $85 \%$ of cases.

A retrospective review was presented of 15 patients with high-grade soft tissue sarcoma who had initially presented with the diagnosis of 'hematoma' [5]. Review was made of the MRI studies, surgical resection and clinical follow-up. Only seven patients in the study had a history of trauma, and only one had undergone anticoagulation. Ninety-three per cent of masses were round or oval, and $67 \%$ lacked peritumoral edema-like changes in signal intensity. Aspiration of the supposed hematomas revealed malignant cytology in only $36 \%$ of cases, with the definitive diagnosis delayed up to 7 months after presentation.

Imaging protocols and techniques

Various improvements in image acquisition were discussed at the annual meeting. In the crusade to preserve image quality yet decrease study acquisition time, two institutions presented papers on isotropic three-dimensional (3D) fast spin echo (FSE) imaging of the extremities, one limited to the rotator cuff $[6,7]$. Comprehensive MR images were acquired of entire joints within 6-7 minutes. When compared with traditional 2D sequences, 3D images tended to be noisier and have more artifacts. However, there remained fair to good agreement and similar confidence among radiologists when comparing 3D with 2D images of the rotator cuff.

Advancement in metal artifact suppression on CT was presented, utilizing Philips' IMC-Plus image-based correction algorithm [8]. This post-processing technique showed improvement in image quality in the immediate vicinity of the metal hardware, and it has the advantage of being applied retrospectively, since it does not require raw image data.

\section{Imaging modalities}

The 44 scientific presentations encompassed many modalities, including radiography, CT, CT arthrography, MR, MR arthrography, sonography and proton MR spectroscopy.
Sonography continues to have an increasing impact on musculoskeletal imaging, and the SSR hosted its first hands-on musculoskeletal ultrasound tutorial located in a collegial setting and attended by many of its members. This instructional event was the highlight of the meeting for many and will hopefully continue at future meetings.

\section{Intervention}

Although the main focus of the meeting was musculoskeletal imaging, musculoskeletal interventional techniques were also addressed. Early experience with percutaneous cryoablation in five patients with extra-abdominal desmoid tumors was presented [9]. One lesion could not be completely ablated due to encasement of the brachial plexus. However, for those patients that were able to be fully treated for their desmoid tumors, percutaneous cryoablation was an effective treatment.

Percutaneous cement augmentation for treatment of sacral and non-sacral pelvic insufficiency fractures in 40 patients was described, with a follow-up of patient-reported pain scales at 24 hours [10]. Although more long-term follow-up is needed for the full understanding of the natural history of treated pelvic fractures, immediate results were promising, and there were no immediate complications.

\section{Normal variation}

Few abstracts addressed investigations into normal imaging variation. The imaging appearance of 236 proximal hamstring origins in symptomatic and asymptomatic individuals was assessed [11]. In 77\% of patients, T1-weighted and T2-weighted signal intensity was increased within the hamstring origins, which did not correlate with symptoms. Four per cent of patients had tuberosity edema, which correlated with physical examination findings, symptoms and a younger age. Four per cent of patients had hamstring tears, which correlated with the patients' symptoms.

One hundred consecutive MRI studies of the wrist were reviewed, demonstrating frequently increased $\mathrm{T} 2$-weighted signal intensity in the pronator quadratus muscles compared with that in the adjacent flexor muscles [12]. The authors postulated that this finding is likely a normal finding and that denervation changes related to anterior interosseous nerve entrapment should not be over-diagnosed.

\section{Radiology education}

The ongoing and future plans of the American Board of Medical Specialties were reviewed by Dr. Tom Berquist, and the Society of Skeletal Radiology is proud to continue providing self-assessment modules (SAMs) for its members in their quest to fulfill their Maintenance of Certification [13]. 
Greater awareness among radiology residents of the American College of Radiology (ACR) musculoskeletal appropriateness criteria was promoted by a study evaluating the familiarity of these criteria, as well as the ability of residents to apply this knowledge within the context of medical decision support [14].

\section{Award-winning papers}

While many paper presentations were noteworthy, two were selected as 'Award Winning.' One will be presented at this year's meeting of the Radiological Society of North America (RSNA), Chicago, IL, USA, and the other at the International Skeletal Society (ISS), Washington, DC, USA. The RSNA award-winning paper was entitled "Contrast enhanced ultrasound characterization of the vascularity of the repaired rotator cuff" by Dr. Kevin Johnson and colleagues from Hospital for Special Surgery in New York [15]. Fourteen patients were evaluated 3-4 months following rotator cuff surgery with contrast-enhanced sonography utilizing microvascular imaging (MVI) software. Contrast-enhanced sonography provided a more sensitive means of evaluating the vascularity of the repaired rotator cuff than did power Doppler, and demonstrated robust vascularity of the suture anchor and peribursal soft tissues, and mild vascularity within the repaired tendon. The ISS award-winning paper was entitled "Temporal evolution of MRI findings after rotator cuff repair" by Christopher J. Hanrahan and colleagues from the University of Utah in Salt Lake City, USA, as previously mentioned [3].

\section{Summary}

As in years past, imaging topics remained the primary focus of the scientific meeting. Additionally, the Society of Skeletal Radiology hosted its first hands-on musculoskeletal ultrasound tutorial, which will hopefully continue at future meetings.

\section{References}

1. Society of Skeletal Radiology. Society of Skeletal Radiology 2009 Annual Meeting. Skeletal Radiol. 2009;38:399-416.

2. White L, Lam B. MR imaging appearance of acute "high ankle sprain" syndesmotic injuries in professional ice hockey players. Skeletal Radiol. 2009;38:409.

3. Hanrahan C, Lance J, Manaster BJ, Burks R, Greis P, Crim J. Temporal evolution of MRI findings after rotator cuff repair. Skeletal Radiol. 2009;38:415.

4. Murphey M, Ruble CH, Jaovisidha S, Temple HT, Gannon F, Jelinek $\mathrm{J}$, et al. Imaging of telangiectatic osteosarcoma with pathologic comparison. Skeletal Radiol. 2009;38:400.

5. Jelinek J, Malawer M, Sternheim A, Ferraro R, Beaman F, Henshaw R, et al. Clinical presentation, outcome and MR findings of soft tissue tumors presenting as hematomas. Skeletal Radiol. 2009;38:399-400.

6. Hill D, Myers R, Lum D, Busse R, Brown B, Tuite M. Isotropic 3D T2-weighted MR images of the rotator cuff at $3 \mathrm{~T}$. Skeletal Radiol. 2009;38:403-4.

7. Deibler A, Childs D, Boles C. Isotropic 3-dimensional fast spin echo imaging of the musculoskeletal system. Skeletal Radiol. 2009;38:403-4.

8. Ramirez I, Northington A, Brown K, Buckwalter K. Metal artifact suppression on $\mathrm{CT}$-preliminary results using a new postprocessing technique. Skeletal Radiol. 2009;38:412.

9. Liu P, Kujak J, Johnson G, Callstrom M. Early experience with percutaneous cryoablation of extra-abdominal desmoid tumors. Skeletal Radiol. 2009;38:413-4.

10. Kapellen P, Beall D, Datir A, Depalma M, Stapp A. Percutaneous treatment of pelvic insufficiency fractures: analysis of 40 consecutive cases. Skeletal Radiol. 2009;38:413.

11. Blankenbaker D, Alsheik N, De Smet A, Lindstrom M. MR appearance of the proximal hamstring origin in symptomatic and asymptomatic individuals. Skeletal Radiol. 2009;38:410 1.

12. Gyftopoulos S, Rosenberg ZS. Increased T2 weighted signal in the pronator quadratus muscle: does it always mean anterior interosseous neuropathy? Skeletal Radiol. 2009;38:405.

13. Berquist T. Maintenance of certification: what you need to know. Skeletal Radiol. 2009;38:402.

14. Logie C, Smith S. Evaluation of resident familiarity and utilization of the ACR MSK appropriateness criteria in the context of medical decision support. Skeletal Radiol. 2009;38:414.

15. Johnson K, Gallo R, Maderazo A, Fealy S, Gamradt S, Warren R, et al. Contrast-enhanced ultrasound characterization of the vascularity of the repaired rotator cuff. Skeletal Radiol. 2009;38:399-416. 